\title{
Novel Functional Genomics Approaches: A Promising Future in the Combat Against Plant Viruses
}

\author{
Vincent N. Fondong, Ugrappa Nagalakshmi, and Savithramma P. Dinesh-Kumar
}

First author: Department of Biological Sciences, Delaware State University, Dover; second author: Department of Plant Biology, College of Biological Sciences, University of California, Davis; and third author: Department of Plant Biology and The Genome Center, College of Biological Sciences, University of California, Davis.

Accepted for publication 28 June 2016.

\begin{abstract}
Fondong, V. N., Nagalakshmi, U., and Dinesh-Kumar, S. P. 2016. Novel functional genomics approaches: A promising future in the combat against plant viruses. Phytopathology 106:1231-1239.

Advances in functional genomics and genome editing approaches have provided new opportunities and potential to accelerate plant virus control efforts through modification of host and viral genomes in a precise and predictable manner. Here, we discuss application of RNAbased technologies, including artificial micro RNA, transacting small

interfering RNA, and Cas9 (clustered regularly interspaced short palindromic repeat-associated protein 9), which are currently being successfully deployed in generating virus-resistant plants. We further discuss the reverse genetics approach, targeting induced local lesions in genomes (TILLING) and its variant, known as EcoTILLING, that are used in the identification of plant virus recessive resistance gene alleles. In addition to describing specific applications of these technologies in plant virus control, this review discusses their advantages and limitations.
\end{abstract}

Plant viruses are widespread in nature and cause devastating diseases in plants, impacting both subsistent and commercial crop production worldwide. The effects of these viruses on plants range from stunted growth, reduced vigor, and decreased marketability of the products to major yield loss. Out of $15 \%$ of the global food production that is lost due to plant diseases, virus diseases account for 47\% of this loss (Anderson et al. 2004; Boualem et al. 2016; Popp and Hantos 2011). Unlike other plant pathogens such as fungi and bacteria, chemical control of plant viruses is not possible. Thus, beyond virus control efforts using cultural practices (Hull 2009), sources of resistance to particular viruses have, in a few cases, been found and successfully incorporated into agriculturally important cultivars by introgression through breeding programs. However, breeding programs are time consuming and, often, important agronomic and commercial traits are lost during genetic crosses. Some of the limitations of conventional breeding could be overcome through direct transfer of genes (genetic engineering) via Agrobacterium tumefaciens-mediated plant transformation technology (Gelvin 2003). Genetic engineering overcomes species barrier issues that are a bottleneck in conventional breeding programs and it significantly reduces the time required for developing new varieties (Galvez et al. 2014).

Thirty years ago, genetic engineering provided a breakthrough in virus control efforts in which the expression of a viral gene in the host plant provided resistance to the corresponding virus (Abel et al. 1986). This approach, referred to as pathogen-derived resistance (PDR), has been used successfully as a control strategy against many different plant viruses (Cillo and Palukaitis, 2014; Galvez et al. 2014). PDR was initially thought to be protein-mediated, whereby the expressed viral protein in the plants disrupted the assembly of the infecting viral protein, leading to resistance. This was elucidated in transgenic tobacco plants expressing the Tobacco mosaic virus

Corresponding authors: V. N. Fondong; E-mail address: vfondong@desu.edu; and S. P. Dinesh-Kumar; E-mail address: spdineshkumar@ucdavis.edu
(TMV) coat protein (CP), which was suggested to cause disassembly of TMV particles in the inoculated plants (Beachy 1999). Similarly, resistance generated from transgenic tobacco plants expressing a defective TMV movement protein (dMP) was explained by competition for the binding sites in the plasmodesmata between the dMP and the wild-type virus-encoded MP (Lapidot et al. 1993). Unlike CP- and MP-mediated resistance, the mechanistic basis of viral replicasemediated resistance is largely due to RNA-mediated resistance but a protein-mediated role cannot be ruled out (Cillo and Palukaitis 2014). Evidence that plants expressing an untranslatable virus $C P$ gene interfered with virus replication led to the discovery of RNA-mediated resistance (Lindbo and Dougherty 1992a, b). Thus, noncoding regions of viral genome, viral sequences in sense or antisense orientation or in double-stranded forms (dsRNAs) were shown to carry out RNA-mediated resistance by degrading viral RNA via the small interfering RNA (siRNA) pathway (Baulcombe 1996a, b; Cillo and Palukaitis 2014; Galvez et al. 2014; Lindbo et al. 1993; Waterhouse et al. 1998; Waterhouse et al. 2001).

The siRNAs derived from processing of dsRNAs in hairpin RNAs (hpRNAs) via RNA interference (RNAi) or RNA silencing guide a nucleotide sequence-specific process that induces mRNA degradation or translation inhibition at the posttranscriptional level and, hence, named posttranscriptional gene silencing or epigenetic modification at the transcriptional level, dependent on RNA-directed DNA methylation (Brodersen and Voinnet 2006; Castel and Martienssen 2013; Vaucheret 2006). Because small RNAs (sRNAs) determine the specificity of the RNAi activities through precise base-pairing recognition of their complementary target RNAs (Ding 2010; Ding and Lu 2011), RNAi has been deployed in virus control efforts. Most recent approaches have used two classes of sRNAs, artificial micro RNAs (amiRNAs) and transacting short interfering RNAs (tasiRNAs), to engineer resistance to RNA and DNA plant viruses (Cillo and Palukaitis 2014; Galvez et al. 2014; Teotia et al. 2016). The potential for practical application of these RNAi approaches has generated great activity in crop improvement.

In the postgenomic era, a reverse genetics approach targeting induced local lesions in genomes (TILLING) has been exploited to engineer virus resistance in crops (Gauffier et al. 2016; Nieto et al. 
2007; Piron et al. 2010). TILLING involves induction of mutations in the plant genome, using classical mutagenesis approaches followed by traditional or high throughput next-generation sequencing based approaches to identify the mutations in the gene of interest (Henikoff et al. 2004; Kurowska et al. 2011; McCallum et al. 2000a, b). Application of TILLING in plant virus control has mainly employed EcoTILLING (Comai et al. 2004), where natural genetic variation in populations is examined to identify new virus resistance alleles or to mine for defective alleles of one or more genes whose gene products interfere with the virus infection cycle (Diaz-Pendon et al. 2004; Gauffier et al. 2016; Nieto et al. 2007; Piron et al. 2010; Robaglia and Caranta 2006; Truniger and Aranda 2009). Another emerging novel strategy for control of plant viruses is the gene-editing approach based on the clustered regulatory interspaced short palindromic repeats (CRISPR)/Cas9 (CRISPR-associated protein 9) system (Chaparro-Garcia et al. 2015; Kennedy and Cullen 2015; Zaidi et al. 2016; Zhang et al. 2015). In this review, we discuss applications of RNA-based functional genomics, EcoTILLING, and CRISPRCas9-based genome-editing strategies in plant virus control efforts. Furthermore, we highlight potential advantages and drawbacks of each strategy.

\section{RNA-BASED FUNCTIONAL GENOMICS TECHNOLOGIES IN PLANT VIRUS CONTROL}

The PDR strategies were first introduced in the mid-1980s and have been extensively used for the development of virus-resistant plants (Cillo and Palukaitis 2014; Galvez et al. 2014). The early PDR applications involved expression of whole genes or long genomic segments of viral pathogens in transgenic plants. However, since the late 1990s the use of RNAi or RNA silencing in conferring resistance to plant viruses has become a method of choice (Cillo and Palukaitis 2014; Galvez et al. 2014). RNAi is an evolutionarily conserved process in a wide variety of eukaryotic organisms (Baulcombe 2005; Voinnet 2009) and is thought to have arisen as an RNA surveillance system, as part of a natural defense mechanism against invasive nucleic acids, including viruses (Ding 2010; Ding and Voinnet 2007). In crop improvement, RNAi has been refined to enable the design of technologies to efficiently target and degrade invading viruses.

The early applications of RNAi involved expression of long inverted repeats of viral genes or genome segments, which upon transcription, form dsRNA molecules with a hpRNA structure. The hpRNA is subsequently cleaved into siRNAs of 21 to 24 nucleotides by the RNase III endonuclease DICER (Bernstein et al. 2001; Fusaro et al. 2006). These siRNAs are incorporated into the RNA-induced silencing complex to guide degradation or translational repression in a sequence-specific manner. RNAi has been extensively used to produce transgenic plants with resistance to diverse viruses (Cillo and Palukaitis 2014; Galvez et al. 2014). The encouraging early results obtained from the use of hpRNA to control plant viruses have been tempered by several disadvantages associated with the approach, including poor stability of the transgene in transformed plants, dependence on the expression levels of the antisense strand, and limited penetration of the silencing sequence to the appropriate viral target. Furthermore, because whole viral genes were used in hpRNA, the risks of recombination leading to emergence of new viruses have raised concerns. More recently, the shortcomings of the hpRNA strategy have at least partially been addressed using amiRNAs and tasiRNA approaches, both of which depend on sRNA molecules.

\section{amiRNAs}

One of the well-studied classes of small RNAs includes miRNAs and their biogenesis starts with the transcription of long primary RNAs (pri-miRNAs), typically by RNA polymerase II. pri-miRNAs are characterized by stem-loop structures consisting of a terminal loop, an upper stem, a duplex of miRNA and its complementary strand, a lower stem, and flanking single-stranded basal segments (Voinnet 2009; Xie et al. 2015b). An RNase III endonuclease, DICERLIKE1 (DCL1) processes the pri-miRNA to precursor miRNAs (premiRNAs) to a mature miRNA. This process involves other proteins, including the dsRNA binding proteins HYPONASTIC LEAVES 1 (HYL1), TOUGH (TGH), and the zinc-finger containing protein SERRATE (Fei et al. 2013; Xie et al. 2015b). miRNAs function in a homology-dependent manner against target mRNAs to, typically, either direct cleavage at highly specific sites or suppress translation; these modes of action depend largely on the complementarity between the miRNA and its target sequences (ValenciaSanchez et al. 2006; Voinnet 2009; Xie et al. 2015b).

Replacing miRNA within the natural miRNA precursor does not affect miRNA biogenesis, as long as the secondary structure of the premiRNA is maintained. Thus, endogenous miRNAs have been replaced with amiRNAs that are complementary to viral transcripts to successfully control plant viruses in model and crop plants (Fahim and Larkin 2013; Khraiwesh et al. 2008; Ossowski et al. 2008; Schwab et al. 2006). For example, in the miR159 precursor, miR159 was replaced with an amiRNA designed from Turnip yellow mosaic virus p69 gene and Turnip mosaic virus (TuMV) HC-Pro coding sequence, and transgenic plants thus obtained exhibited strong virus resistance (Niu et al. 2006). Since these encouraging results, resistance to at least 12 plant viruses has been generated, using different miRNA precursors as backbone (Table 1). These include replacing miR159 with amiRNA derived from Cucumber mosaic virus (CMV) 2b, 2a. and 3' untranslated region. Transgenic tomato, Arabidopsis thaliana, and Nicotiana tabacum containing these constructs were resistant to target viruses (Duan et al. 2008; Qu et al. 2007; Zhang et al. 2011). The approach has also been deployed in engineering resistance to Potato virus $Y$ (PVY) by inserting amiRNAs from $\mathrm{CI}$, NIa, NIb, CP, and HC-Pro into four different miRNA precursors (Table 1) (Ai et al. 2011; Song et al. 2014). Correspondingly, amiRNAs from Potato virus $X$ p25 was shown to confer resistance to the virus (Ai et al. 2011). Other viruses targeted, using amiRNAs, include Tobacco etch virus (Song et al. 2014), Grapevine fanleaf virus (Jelly et al. 2012), Watermelon silver mottle virus (Kung et al. 2012), Wheat streak mosaic virus (WSMV) (Fahim et al. 2012), Wheat dwarf virus (WDV) (Kis et al. 2015), Cotton leaf curl Burewala virus (Ali et al. 2013), Tomato spotted wilt virus (Mitter et al. 2016), and Cassava brown streak virus (CBSV) and Uganda CBSV (Wagaba et al. 2016).

The amiRNA platform has several advantages over the hpRNA strategy. These include the fact that amiRNAs are small and, thus, the likelihood of off-target effects is reduced considerably, compared with hpRNA. Also, the small size of amiRNAs permits transgene multiplexing via use of polycistronic miRNA backbone more feasible, and recombination between amiRNA transgene and cognate viruses leading to emergence of new viruses is unlikely. Moreover, processing of miRNA is not affected by changes in temperature compared with hpRNA-derived siRNAs whose levels decrease at low temperatures (Szittya et al. 2003).

A major drawback of the amiRNA strategy is that, because of the small size of the amiRNA ( $21 \mathrm{nt})$, there are increased opportunities for the virus to evolve and escape surveillance due to loss of complementarity with amiRNA, resulting in loss of resistance (Lin et al. 2009). Furthermore, the natural diversity in the virus population may contain mismatches at the amiRNA-binding site and such variants would not bind to the amiRNA and, therefore, can escape surveillance. To reduce these risks, a multimeric amiRNA approach in which multiple amiRNAs targeting different conserved regions of the viral genome has been adopted in several instances (Fahim et al. 2012; Kung et al. 2012; Zhang et al. 2011). This strategy requires a polycistronic precursor miRNA backbone; such is the case of the miR395 precursor gene, which was used to express five amiRNAs targeting different conserved structural and functional portions of the WSMV genome (Fahim et al. 2012). Transgenic 
plants expressing the modified miR395 precursor construct were shown to exhibit a robust resistance to the virus. Similarly, the polycistronic precursor of miR171 was used to produce three amiRNAs that target conserved segments in WDV (Kis et al. 2015). In each of these cases, the arms of the polycistronic miRNA precursor are replaced with different amiRNAs from different regions of the virus.

\section{tasiRNA}

Noncoding TAS genes that have been identified in all examined land plants produce tasiRNAs. TAS genes differ from most other genes in that they do not code for a protein but, rather, produce long noncoding RNA transcripts, which are subsequently processed into 21-nt tasiRNAs. Synthesis of tasiRNA is initiated by miRNAdirected and argonaute (AGO) protein-mediated cleavage of TAS transcripts, of which four (TAS1, TAS2, TAS3, TAS4) have been identified in Arabidopsis (Allen and Howell 2010; Yoshikawa, 2013). Two models of tasiRNA biogenesis, referred to as "one-hit" and "two-hit", have been described in Arabidopsis (Fei et al. 2013). In the one-hit model, where there is a single miRNA target site, a 22-nt miRNA, upon binding to the target, guides cleavage of the target transcript between the 10th and 11th nucleotides of the miRNA, under the control of AGO1 (Chen et al. 2010; Cuperus et al. 2010). In the two-hit model, two miRNA target sites are used during biogenesis; however, 21-nt miRNA-directed AGO7 cleavage of the target occurs only on the 3' target site and the 5' site is required but not cleaved (Axtell et al. 2006). In both models, SGS3 stabilizes cleavage products, which are processed into dsRNA by RDR6. DCL4 then cleaves the dsRNA fragment into phased 21-nt dsRNA registers downstream from the cleavage site for the one-hit model and upstream from the $3^{\prime}$ cleavage site for the two-hit model (Allen and Howell 2010). In both one-hit and two-hit models, one strand of the resulting 21-nt duplex targets and degrades the corresponding mRNA in trans.

The tasiRNA strategy has been used to successfully engineer resistance to plant viruses. In this platform, the TAS gene is modified to contain viral sequences downstream of the miRNA cleavage site for the one-hit model or upstream of the cleavage site for the two-hit model (Fig. 1). Thus, transgenic Nicotiana benthamiana harboring a TAS3 gene, modified to contain tasiRNA from the AC2 and AC4 genes of the geminivirus Tomato leaf curl New Delhi virus (ToLCNDV), exhibited strong resistance to ToLCNDV and Tomato leaf curl Gujarat virus (Singh et al. 2015). Also, transgenic A. thaliana plants expressing TAS3 modified with tasiRNAs from TuMV and CMV were highly resistant to both viruses (Chen et al. 2016). Furthermore, transgenic $N$. benthamiana containing Arabidopsis TASla gene modified with tasiRNA from the cassava geminivirus East African cassava mosaic Cameroon virus exhibits strong resistance to the virus (V. Fondong, U. Nagalakshmi, S. P. Dinesh-Kumar, B. Khatabi, R. Xia, B. C. Meyers, and V. N. Fondong, unpublished). These findings emphasize the potency of this strategy in plant virus control.

There are many advantages associated with the use of tasiRNA in plant virus control. First, unlike the hpRNA strategy, tasiRNAs as short as $21 \mathrm{nt}$ may be sufficient to silence genes and, thus, sequences from multiple targets can be stacked in one tasiRNA construct, making the approach easily amenable to multiplexing. Furthermore, unlike hpRNA and the amiRNA strategies that require folding into a dsRNA structure, tasiRNAs are processed from singlestranded RNA (ssRNA) and, thus, structural considerations are not critical. Additionally, systemic spread of tasiRNA throughout a plant is more efficient than amiRNA or hpRNA-derived siRNA (Felippes and Weigel 2009), thus, tasiRNAs will more efficiently silence targets in a constitutive manner. Also, tasiRNA selectively silences target genes without toxicity or off-target silencing. Moreover, since short sequences are used in this strategy, complementation or recombination of transgenes with related viruses, resulting in emergence of more virulent viruses, is unlikely when compared with the hpRNA approach. Thus, the tasiRNA strategy has significant promise as a virus control tool.

The main disadvantage of use of tasiRNAs in plant improvement is that this approach requires coexpression of exogenous miRNAs, $T A S$ genes, and their promoters; this is principally because current applications use well-studied Arabidopsis TAS genes. However, as $T A S$ genes and their miRNA triggers are discovered and characterized in respective crop plants, these endogenous $T A S$ genes can be modified to express viral sequences, which would be the only transgene in the construct.

\section{TILLING AND EcoTILLING APPROACHES}

TILLING is a nonrecombinant reverse genetics approach that combines traditional mutation induction with high throughput mutation discovery (McCallum et al. 2000a, b). In TILLING, the seeds are treated with a chemical mutagen such as ethylmethanesulfonate (EMS), which primarily introduces $\mathrm{G} / \mathrm{C}$ to $\mathrm{A} / \mathrm{T}$ transitions, and M1 plants thus obtained are self-fertilized to generate M2 individuals (McCallum et al. 2000a, b). The M2 plants are used to

TABLE 1. Artificial micro RNAs (miRNAs) used to engineer resistance to plant viruses ${ }^{\mathrm{a}}$

\begin{tabular}{|c|c|c|c|c|}
\hline miRNA precursor backbone & Plant species & Virus/gene targeted & Virus family/genome & References \\
\hline $\operatorname{miR} 159$ & Arabidopsis thaliana & Turnip mosaic virus/p69, Hc-Pro & Potyviridae/+ssRNA & $\begin{array}{l}\text { Lin et al. } 2009 \\
\text { Martínez et al. } 2013 \\
\text { Niu et al. } 2006\end{array}$ \\
\hline $\operatorname{miR} 159$ & A. thaliana & Turnip yellow mosaic virus/P69 & Tymoviridae/+ssRNA & Niu et al. 2006 \\
\hline $\operatorname{miR} 159 a$ & $\begin{array}{l}\text { Tomato } \\
\text { A. thaliana } \\
\text { Nicotiana tabacum }\end{array}$ & Cucumber mosaic virus/2a, 2b, 3' UTR & Bromoviridae/+ssRNA & $\begin{array}{l}\text { Duan et al. } 2008 \\
\text { Qu et al. } 2007 \\
\text { Zhang et al. } 2011\end{array}$ \\
\hline $\operatorname{miR} 159 a$ & N. benthamiana & Watermelon silver mottle virus/L & Bunyaviridael-ssRNA & Kung et al. 2012 \\
\hline $\operatorname{miR} 159 a$ & N. benthamiana & Tomato spotted wilt virus/N, NSs & Bunyaviridae/-ssRNA & Mitter et al. 2016 \\
\hline $\operatorname{miR} 159 a$ & $N$. benthamiana & $\begin{array}{l}\text { Cassava brown streak virus (CBSV) and } \\
\text { Uganda } \mathrm{CBSV} / \mathrm{P} 1, P 3, C I, N I b, C P \text {, and } \\
\text { 3' UTR }\end{array}$ & Potyviridae/+ssRNA & Wagaba et al. 2016 \\
\hline $\mathrm{miR} 159 \mathrm{a} / \mathrm{miR} 167 \mathrm{~b} / \mathrm{miR} 171 \mathrm{a}$ & N. tabacum & Potato virus $X / \mathrm{p} 25$ & Alphaflexiviridae/+ssRNA & Ai et al. 2011 \\
\hline $\operatorname{miR} 169 a$ & N. benthamiana & Cotton leaf curl Burewala virus/V2 & Geminiviridae/ssDNA & Ali et al. 2013 \\
\hline $\operatorname{miR} 171$ & Barley & Wheat dwarf virus/Rep and MP & Geminiviridae/ssDNA & Kis et al. 2015 \\
\hline miR319a/ miR159a/ & & & & Ai et al. 2011 \\
\hline $\mathrm{miR} 167 \mathrm{~b} / \mathrm{miR} 171 \mathrm{a}$ & N. benthamiana, N. tabacum & Potato virus $\mathrm{Y} / \mathrm{CI}, \mathrm{NIa}, \mathrm{NIb}, \mathrm{CP}, \mathrm{Hc}-$ Pro & Potyviridae/+ssRNA & Song et al. 2014 \\
\hline $\operatorname{miR} 319 a$ & N. benthamiana & Tobacco etch virus/CI, NIa, NIb, CP & Potyviridae/+ssRNA & Song et al. 2014 \\
\hline $\operatorname{miR} 319 a$ & Grapevine somatic embryos ${ }^{b}$ & Grapevine fanleaf virus/CP & Secoviridae/+ssRNA & Jelly et al. 2012 \\
\hline $\operatorname{miR} 395$ & Wheat & Wheat streak mosaic virus/genome-wide & Potyviridae/+ssRNA & Fahim et al. 2012 \\
\hline
\end{tabular}

${ }^{\text {a }}$ UTR = untranslated region; NSs = nonstructural protein; $\mathrm{CP}=$ coat protein; ssRNA = single-stranded RNA

b Transient expression. 
prepare DNA samples for mutational screening, while an inventory of their seeds is established for future and downstream research. DNA samples are then pooled to maximize the efficiency of mutation detection. Polymerase chain reaction (PCR) is performed using $5^{\prime}$ end-labeled gene-specific primers to target the desired locus, followed by heating and cooling of the PCR product to form DNA heteroduplexes, which are cleaved with CEL I nuclease, a plant-specific extracellular glycoprotein that preferentially cleaves mismatches of all types (Oleykowski et al. 1998). The cleaved products representing induced mutations are visualized with denaturing polyacrylamide gel electrophoresis and mutants are identified. This procedure has successfully been employed to identify gene mutations in several plant species (Guo et al. 2015; Kurowska et al. 2011). Advances in sequencing, especially with the advent of high-throughput sequencing, have greatly accelerated application of TILLING in functional genomics (Guo et al. 2015; Tsai et al. 2011, 2015).

EcoTILLING, an adaptation of the TILLING, is used in detecting rare single nucleotide polymorphism (SNPs) or small INDELs in target genes in natural populations (Comai et al. 2004). In EcoTILLING, mismatches formed by hybridization of different genotypes in a test panel are cleaved with CEL I. A valuable application of EcoTILLING in plants is in the search of variation in disease resistance genes. Thus, this procedure was used to identify recessive virus-resistance genes, a typical example being virus-resistant alleles of the eukaryotic translation initiation factor 4F (eIF4F) gene family, which has two subunits: eIF4E and eIF4G (Robaglia and Caranta 2006; Truniger and
Aranda 2009). It is important to indicate that almost all known plant genes with natural or induced recessive resistance alleles encode eIF4E or eIF4G gene families (Diaz-Pendon et al. 2004; Robaglia and Caranta 2006; Truniger et al. 2008). Also, EcoTILLING was successfully used to identify a nonsilent allelic variant of eIF4E from 113 EcoTILLING accessions of melon, and the variant was observed to confer resistance to Melon necrotic spot virus (Nieto et al. 2007). Correspondingly, Ibiza et al. (2010) identified five new resistance alleles of eIF4E that exhibited resistance to PVY in Capsicum spp. Additionally, a splicing mutant of eIF4E from an EMS-mutagenized population was found to be immune to two strains of PVY and Pepper mottle virus (Menda et al. 2004). These reports indicate that EcoTILLING has great promise as an approach to accelerate identification of natural virus disease resistance alleles.

TILLING and EcoTILLING approaches have several advantages over other reverse genetics strategies. Importantly, unlike other routinely used approaches, such as insertional mutagenesis and RNAi, it does not rely on transgenic plants and would, therefore, be a method of choice for transformation of recalcitrant plant species. Also, TILLING is highly efficient in causing large numbers or a high density of mutations in a plant genome. Furthermore, gene regions are targeted for mutation discovery with no bias, as products of PCR amplification are used for screening (Wang and Shi 2015). EcoTILLING does not require burdensome, expensive, and timeconsuming sequencing of all individuals in a population to identify

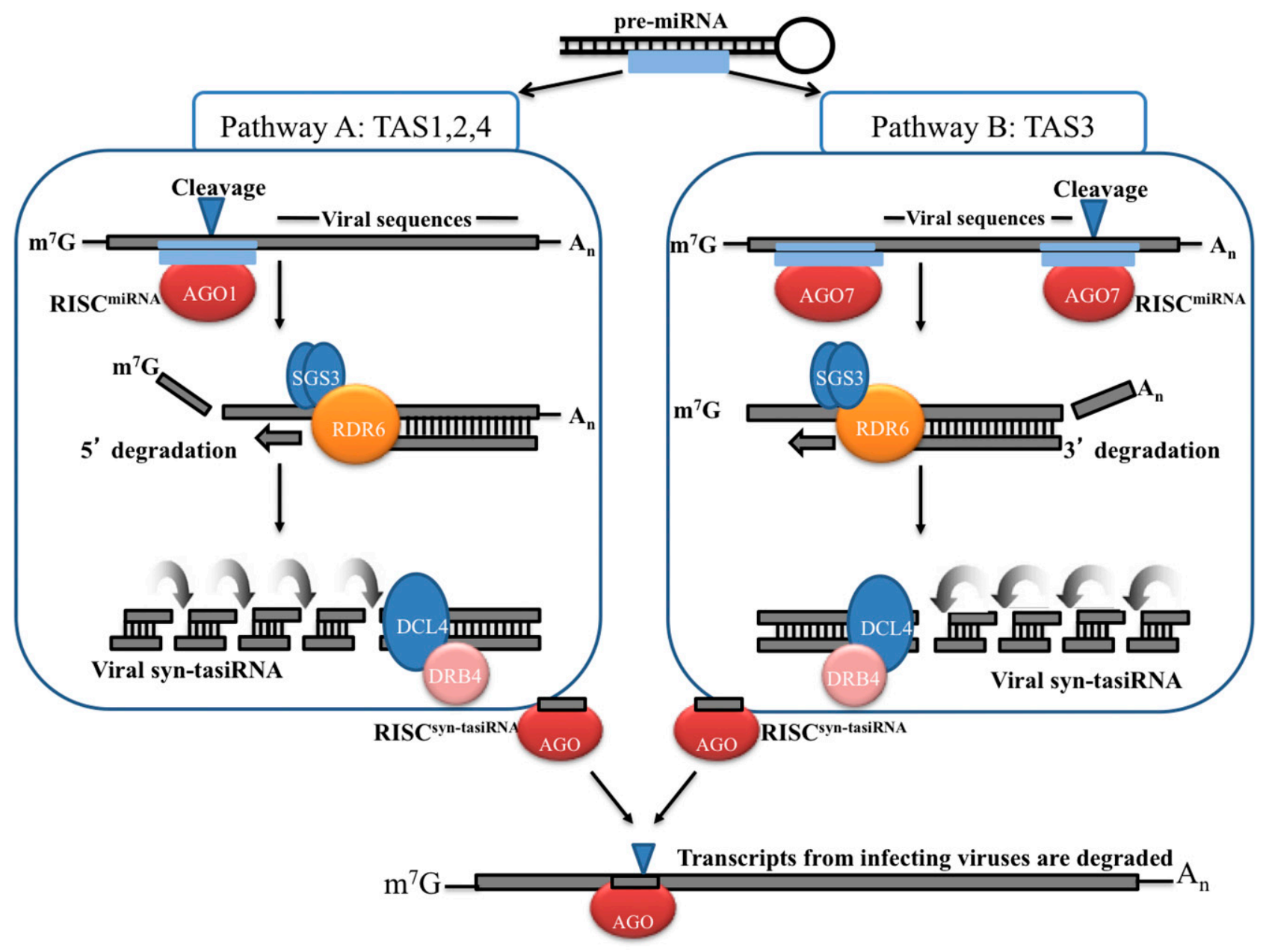

Fig. 1. Two transacting small interfering RNA (tasiRNA) pathways are known in Arabidopsis. In pathway A ("one-hit"), micro RNA (miRNA) guided cleavage occurs at the 5' end of the transcript. In pathway B ("two-hit"), miRNA binding occurs at two sites, with cleavage only at the 3' site (adapted from Allen and Howell [2010]). The TAS gene is modified to contain sequences from the virus or viruses being targeted and, upon processing, phased tasiRNAs are produced; these tasiRNAs direct the silencing complex to transcripts from cognate invading viruses. 
polymorphisms (Barkley and Wang 2008), and it detects multiple polymorphisms in a single fragment, given that CEL I digests only a small proportion of the heteroduplexes at a single position (Till et al. 2006). Additionally, unlike other SNP and INDEL detection methods, TILLING provides the approximate location of SNPs or INDELs in the target gene from a mutated population (Barkley and Wang 2008; Henikoff et al. 2004; Ülker and Weisshaar 2010). Moreover, because TILLING produces different kinds of mutations (nonsense: 5\%; missense: 65\%; silent: 30\%) (McCallum et al. 2000a), it produces alleles with partial or complete loss of function as well as alleles with novel functions, all of which can provide valuable insights into gene function (Ülker and Weisshaar 2010).

The disadvantage of TILLING and EcoTILLING is the requirement for locus-specific PCR products, which would be difficult for gene families with very similar sequences and in polyploids. Additionally, it is not possible to detect mutations near simple sequence repeats due to polymerase slippage-induced deletions (Ülker and Weisshaar 2010).

\section{GENOME EDITING APPROACHES}

Targeted genome editing strategies have become efficient methods for inducing targeted deletions, insertions, and precise sequence changes in genomes. Until recently, genome editing approaches such as zinc finger nucleases (ZFNs) and transcription-activatorlike effector nucleases (TALENs) were the methods used in introducing site-specific mutations in targeted genes (Baltes and Voytas 2015; Kim and Kim 2014; Voytas 2013). Generating an effective ZFN or TALEN is labor-intensive, making practical application of these methods in plant virus control challenging. Thus, the recent emergence of the CRISPR and Cas protein system as a facile and efficient alternative to ZFNs and TALENs for inducing targeted genetic alterations has provided new opportunities in crop improvement, including plant virus control. The ease of deployment of the CRISPRCas system is due to its dependence on RNA as the moiety that targets the Cas nuclease to a desired DNA sequence (Sander and Joung 2014; Sternberg and Doudna 2015; Wright et al. 2016).

In bacteria, especially in archaea, the CRISPR-Cas system confers molecular immunity to foreign nucleic acids, including plasmids and viruses (Barrangou 2015; Barrangou and Marraffini 2014). In the CRISPR-Cas system, CRISPR RNAs direct Cas endonucleases to cleave invading nucleic acids on the basis of sequence complementarity (Bhaya et al. 2011). CRISPR genomic loci consist of repeat sequences, typically 20 to $50 \mathrm{bp}$ in length, separated by variable spacer sequences (or protospacers) of similar length that match a segment of invading nucleic acids. These protospacers serve as a molecular memory of prior infections and, together with repeat sequences, constitute CRISPR RNAs in the CRISPR locus (Barrangou 2015; Barrangou and Marraffini 2014). CRISPR RNAs are used as molecular guides by Cas proteins for base-pairing with and degradation of complementary sequences in invading DNAs (Barrangou 2015; Barrangou and Marraffini 2014). The Cas9 endonuclease has two domains, $\mathrm{HNH}$ and RuvC, which cleave the target DNA $3 \mathrm{nt}$ upstream of the protospacer adjacent motif (PAM) (Jinek et al. 2012) (Fig. 2).

Evidence that the CRISPR-Cas system is functional in eukaryotic systems has revolutionized genome editing in plants, for which the Streptococcus pyogenes endonuclease Cas9 (SpCas9) has been harnessed for efficient genome editing and gene regulation (Cong et al. 2013; Haurwitz et al. 2010; Jinek et al. 2013). To simplify the engineering and target specificity of the CRISPR-Cas9 system, the repeat sequences and protospacer have been combined into a single chimeric guide RNA (sgRNA) molecule that is functionally expressed under small nuclear RNA promoters such as U6 or U3 (Cong et al. 2013; Jinek et al. 2013) (Fig. 2). Many studies have elucidated successful and effective application of this system in plant genome editing (Baltes and Voytas 2015; Belhaj et al. 2015; Liu et al. 2016).

In addition to direct targeting of the viral genome using the CRISPR-Cas9 system, several other CRISPR-Cas9 platforms have been described, including nontransgenic delivery of CRISPRCas/sgRNA and CRISPR-Cas9 gene-driven modification of insect virus vector populations; these platforms are discussed below.

\section{DIRECT TARGETING OF VIRAL GENES AND MODIFICATION OF VIRAL HOST FACTORS USING CRISPR-Cas9}

Recent reports have shown that the CRISPR-Cas9 system can target plant DNA viruses and confer virus resistance. For example, a CRISPR-Cas9 containing sgRNA designed from the Rep and CP genes as well as the intergenic region of the two geminiviruses Tomato yellow leaf curl virus (TYLCV) and Beet curly top virus (BCTV) targeted and degraded both viruses in $N$. benthamiana plants expressing Cas9 in a virus-specific manner (Ali et al. 2015b). These authors further showed that a single sgRNA containing the invariant TAATATTAC nonanucleotide sequence found in the origin of replications of all geminiviruses, targeted and degraded TYLCV, BCTV, and Merremia mosaic virus, suggesting a broadspectrum resistance against geminiviruses. Accordingly, sgRNAs designed from diverse coding and noncoding regions of another geminivirus, Beet severe curly top virus (BSCTV), was shown to specifically target and degrade BSCTV in transgenic Arabidopsis and $N$. benthamiana (Ji et al. 2015). Similar results were obtained by Baltes et al. (2015), who tested sgRNA from the Bean yellow dwarf virus (BeYDV) Rep-binding site, hairpin, nonanucleotide sequence, and three Rep motifs essential for rolling circle replication. This report further observed that $\operatorname{sgRNAs}$ targeting sequences near the BeYDV hairpin were less effective, presumably due to formation of a secondary structure that impeded Cas9-sgRNA cleavage or access (Baltes et al. 2015).

All studies described to date in plants utilized SpCas9 to target DNA geminiviruses. However, recently, Cas9 from Francisella novicida, which targets RNA instead of DNA, was engineered to target and inhibit hepatitis $\mathrm{C}$ virus, a positive-sense ssRNA virus, within eukaryotic cells (Price et al. 2015). This shows that FnCas9 can effectively function in eukaryotic cells and be programmed as

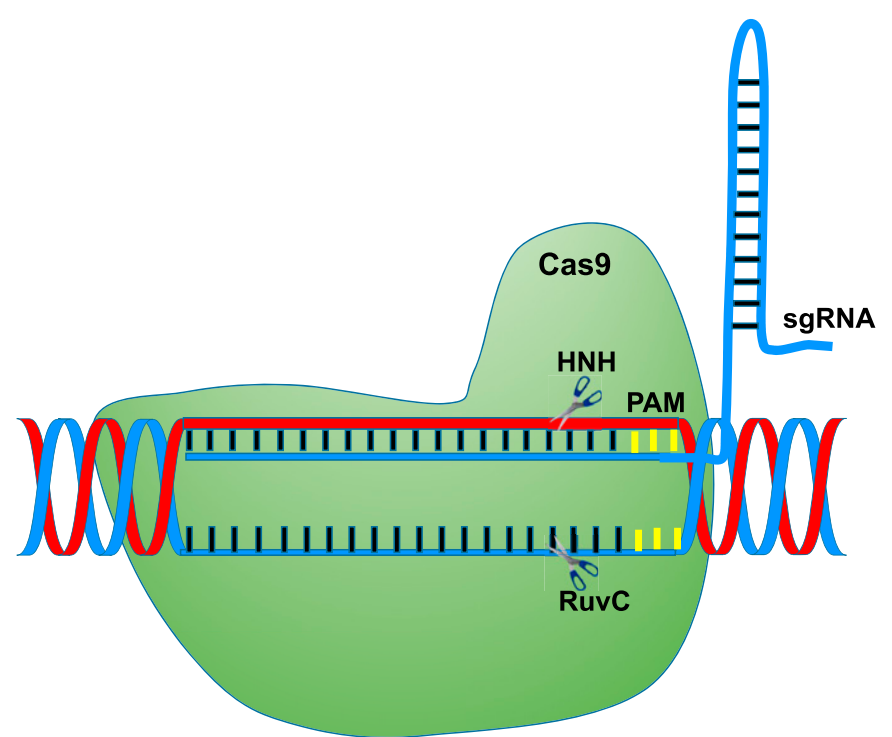

Fig. 2. The Cas9/single chimeric guide RNA (sgRNA) ribonuclease. In this system, the clustered regularly interspaced short palindromic repeat RNA of the sgRNA guides Cas9 and binds with the complementary target sequence, thereby determining target specificity. The presence of a protospacer-adjacent motif (PAM) directly downstream from the target DNA is a prerequisite for DNA cleavage by Cas9. The nuclease part has two nuclease domains ( $\mathrm{HNH}$ and RuvC). Cas9 and the sgRNA form a dual ribonuclease complex capable of binding the complementary strand of the target site and creating a doublestranded break three bases upstream of the PAM. 
defense against RNA viruses. Because most plant viruses have an RNA genome, the discovery of FnCas9 greatly expands the scope of application of CRISPR-Cas to engineer virus resistant crops. RNAguided RNA targeting Class 2 type VI-A CRISPR-Cas effector $\mathrm{C} 2 \mathrm{c} 2$ has recently been shown to cleave ssRNA and mRNAs in bacteria (Abudayyeh et al. 2016). In the future, C2c2 could be engineered to target RNA viruses.

The use of CRISPR-Cas9 system in the control of plant RNA viruses through modification of a host factor required for potyvirus infection cycle in transgenic cucumber and Arabidopsis was recently elucidated (Chandrasekaran et al. 2016; Pyott et al. 2016). In these studies, sgRNA was designed to target alleles of $e I F 4 E$ or $e I F(i s o) 4 E$, two genes that are essential for the translation of viral RNA genomes in which VPg, a viral protein, is covalently linked to the $5^{\prime}$ end (Wittmann et al. 1997). Elongation factors eIF4E and eIF(iso)4E interact with VPg in different hosts, and disruption of this interaction by mutagenesis or silencing prevents virus infectivity completely (Jiang and Laliberte 2011). It was shown that transgenic cucumber plants containing Cas9-sgRNA constructs targeting the eIF4E gene produced small deletions and SNPs in the eIF4E and the homozygous progeny plant lines exhibited immunity to Cucumber vein yellowing virus, Zucchini yellow mosaic virus, and Papaya ring spot mosaic virus (Chandrasekaran et al. 2016). Similarly in Arabidopsis, CRISPR-Cas9 mediated modification in eIF(iso)4E exhibited complete resistance to TuMV (Pyott et al. 2016). In addition, the homozygous mutation in eIF(iso)4E had no effect on the overall plant growth phenotypes. These findings provide an unprecedented opportunity in the design of broad-spectrum control for plant viruses whereby sgRNA can be engineered to knock out or modify host genes required in the virus infection cycle.

Recently, a robust multiplexing strategy for the CRISPR-Cas9 system was described, whereby the guide RNA was engineered as a single polycistronic gene based on endogenous transfer (t)RNAprocessing system (Xie et al. 2015a). The synthetic polycistronic gene consists of tandemly arrayed tRNA-sgRNA units, with each unit consisting of a conserved tRNA and a sgRNA containing a targetspecific spacer and conserved sgRNA scaffold. Upon transcription, the tandemly arrayed tRNA-sgRNA chimera is cleaved by endogenous RNase $\mathrm{P}$ and RNase $\mathrm{Z}$ to release tRNA and the mature sgRNAs, which direct Cas9 to multiple targets. Because tRNA and its processing system are virtually conserved in all living organisms, it was suggested that this method could be used to simultaneously target multiple genes (Xie et al. 2015a). Thus, this multiplex strategy along with SpCas9 and FnCas9 could be used to target either multiple plant RNA and DNA viruses, multiple genes in a single virus, or both.

\section{NONTRANSGENIC DELIVERY OF CRISPR-Cas/sgRNA}

Although the CRISPR-Cas9 transgene could be segregated away once the induced desired mutation is identified in transgenic plants, it is unclear whether the regulatory authorities consider these plants as free of genetic modification. In this regard, preassembled Cas9 protein-sgRNA ribonucleoproteins (RNP), rather than plasmids that encode these components, have been used to induce targeted genome modifications in different plant species and, in each case, the mutations induced were stably maintained in whole plants that were regenerated from protoplasts (Woo et al. 2015). Because no recombinant DNA is used in this process, the resulting genomeedited plants might be exempt from current genetically modified organism (GMO) regulations (Kanchiswamy et al. 2015), paving the way for use of this approach without restrictions that may be imposed on stably transformed plants by regulatory organizations.

Plant virus-based expression vectors can also be engineered to deliver Cas9 and sgRNAs to induce mutations in a GMO-free manner. Since plant RNA viruses do not integrate into the plant genome, they are not transmitted through germline mutation to the next generation; the induced mutation will be free of transgenes.
Recently, Tobacco rattle virus-based RNA viral vector was used to deliver sgRNA targeted to the PDS (phytoene desaturase) gene in $N$. benthamiana plants expressing Cas9 (Ali et al. 2015a). Although the efficiency of germline transmission is relatively low in this study, it is possible to optimize the vector to enhance the frequency of transmission of mutations to the next generation. Modification of this viral expression system may also facilitate codelivery of Cas9 and $\operatorname{sgRNAs}$ into plants to modify host factors required for the virus infection cycle to generate non-GMO plants. Furthermore, one could envision delivery of Cas9 and sgRNAs targeted to the virus genes or genome using attenuated viral expression vectors as a virus disease-control strategy. However, the means of delivery of these engineered attenuated viruses needs to be worked out for field applications. Compared with a preassembled Cas9-RNP system, the viral vector-based delivery system bypasses the tissue culture regeneration step that often could induce undesirable modifications in the genome. Also, a wide number of plant viruses have been engineered as expression vectors that can work in diverse hosts in different families.

\section{ENGINEERING PLANT VIRUS RESISTANCE USING CRISPR-Cas9 GENE-DRIVEN MODIFICATION OF INSECT VECTOR POPULATIONS}

Gene drives are naturally existing genetic elements that function by increasing in frequency with each generation, even without conferring a fitness advantage upon their host, thus forcing nonMendelian inheritance patterns (Champer et al. 2016; Esvelt et al. 2014). Such inheritance-bias, which is a common strategy in nature (Burt and Trivers 2009), may spread by cutting homologous chromosomes that do not contain the gene drive and inducing cellular repair to copy the drive onto the damaged chromosome by homologous recombination (Champer et al. 2016). The use of gene drives to induce biased inheritance of specific genes to spread desirable traits through wild populations has been reported (Burt 2014; Champer et al. 2016; Esvelt et al. 2014). Although engineering of gene drives based on homing endonuclease genes have been proposed (Burt 2003), the application of this procedure has been hindered by technical challenges (Champer et al. 2016; Esvelt et al. 2014). However, the discovery of CRISPR-Cas9 has provided new opportunities in the application of this approach to control vector-borne diseases (Adelman and Tu 2016). In this approach, the gene drive is designed so that there is homology between sequences flanking the drive element and the targeted locus for the cell to use homologous recombination to incorporate the gene drive. Thus, the CRISPR-Cas9 gene drive system consists of a cassette carrying a Cas9 transgene, an sgRNA targeting a genomic sequence of interest, and flanking homology arms corresponding to genomic sequences abutting the target cleavage site. Upon cleavage, the core Cas9/sgRNA cassette is inserted into the targeted locus via homology-directed repair and, in turn, the inserted cassette expresses both Cas9 and the sgRNA, leading to cleavage and homologymediated insertion of the cassette into the second allele, thereby rendering the mutation homozygous (Gantz and Bier 2015). This strategy may be particularly useful for the control of vector-borne plant viruses, as has been demonstrated for Anopheles spp. vectors of malaria and dengue fever (Gantz and Bier 2015; Hammond et al. 2016; Kistler et al. 2015).

Although gene drive applications have not been employed in plant virus control, for example, by targeting the insect vectors that transmit many plant viruses, the CRISPR-Cas9 gene drive system can be designed to inhibit the ability of the insect populations to acquire and transmit viruses. This can be achieved either by targeting insect vector genes involved in acquisition and transmission of the virus or by modifying genes involved in sex determination in favor of male insects or genes that influence female fecundity. The spread of a gene drive through a target population would depend on molecular factors, including homing efficiency, fitness cost, and evolutionary 
stability (Marshall and Hay 2012), while the rate of spread is determined by the mating dynamics, generation time, and other characteristics of the target population (Esvelt et al. 2014).

\section{IMPACT OF THE EMERGING CRISPR-Cas9 APPROACH ON PLANT VIRUS DISEASE CONTROL}

The discovery of CRISPR-Cas9 system has facilitated the deployment of easy and programmable platforms for genome editing and transcriptional control in a sequence-specific manner. This is largely due to several advantages of this approach over other genome editing and functional genomics strategies. For example, construction of CRISPR is simple compared with ZFN and TALEN, especially because DNA recognition in CRISPR is via nucleic acid (sgRNA) rather than protein, as in ZFN and TALEN; thus, cloning of the DNA-recognition component of sgRNA is simple (Chandrasegaran and Carroll 2016). Correspondingly, the CRISPR nucleotide-directed specificity system is much easier to customize and, thus, provides flexibility. Furthermore, the small size of sgRNAs (18 to $20 \mathrm{nt}$ ) makes construction of vectors with multiple guide RNAs for multiplexed gene targeting simple, compared with ZFNs and TALENs. Finally, CRISPR is not sensitive to methylation and does not require an additional a priori determination of the methylation state of the target site as found in TALEN and ZFN approaches.

There are some limitations in the CRISPR-Cas9 system. For instance, CRISPR sgRNA targets must immediately precede a PAM site; while it is usually not difficult to locate these sites in the target, this constraint sometimes causes problems for specific applications. The requirement of PAM contrasts with TALENs, for which, although the design requires offset binding proteins with defined spacing, no design constraints have been described. Thus, in principle, a TALEN pair can be targeted to any site in a genome, providing more flexibility compared with CRISPR. In functional genomics approaches, such as hpRNA, tasiRNA, and amiRNA, targets are mostly repressed, whereas CRISPR-Cas9 knocks out and abolishes the target genes leading to increased penetrance of the phenotype. In RNAi, the RNA guides can be extracted directly from invading nucleic acids; thus, the capacity for mutational evasion by the invader is limited. In contrast, CRISPR sequence determinants must first become encoded in the host genome before they are accessible in defense; thus, targets have greater potential for mutational evasion in the CRISPR approach. Also, because the sgRNA in CRISPR tolerates, to varying degrees, up to five mismatches (non-Watson-Crick base pairing) with unwanted target sites (Fu et al. 2013), CRISPR may be more prone to off-targeting compared with TALEN (Kuscu et al. 2014). The use of paired single-strand break (nickase) mutants, which require targeting two guide RNAs on opposite strands flanking the target site has been shown to reduce CRISPR-Cas9 off-targeting (Mali et al. 2013; Ran et al. 2013). The efforts in improving the specificity of CRISPR are focused on rational design of sgRNA, selection of eligible CRISPR nuclease, choice of suitable target sites, and delivery of Cas9-sgRNA into cells followed by rapid degradation of the nuclease (Jamal et al. 2015). Because of the rapid pace with which the CRISPR-Cas9 system is being evaluated for off-targeting and other applications, this technology will have a major impact on future control of plant virus diseases.

\section{CONCLUSION}

Currently, the only successful example of transgenic virus-resistant crop consumed by humans includes the PDR-based Papaya ringspot virus-resistant Rainbow variety of papaya (Gonsalves 1998). We anticipate that, in the postgenomic era, there are clear potentials for augmenting engineering host resistance to viruses using amiRNA, tasiRNA, TILLING/EcoTILLING, and CRISPR-Cas9 technologies. The increasing reduction in the cost of high-throughput sequencing and lessons from ongoing and past work will continue to provide new insights into these and additional new genome editing and functional genomic approaches. Because of increasing incidents of mixed infections by multiple viruses as well as the emergence of virus variant populations, there will be a need to multiplex these strategies to engineer broad-spectrum virusresistant crops.

\section{ACKNOWLEDGMENTS}

The disease resistance work in the V. Fondong laboratory is supported by the National Science Foundation Integrative Organismal Systems (NSFIOS) grant NSF-IOS-1212576 and in the S. P. Dinesh-Kumar laboratory is supported by National Institutes of Health grant NIH-R01GM097587 and NSF-IOS-1354434 and NSF-IOS-1339185.

\section{LITERATURE CITED}

Abel, P. P., Nelson, R. S., De, B., Hoffmann, N., Rogers, S. G., Fraley, R. T., and Beachy, R. N. 1986. Delay of disease development in transgenic plants that express the tobacco mosaic virus coat protein gene. Science 232:738-743.

Abudayyeh, O. O., Gootenberg, J. S., Konermann, S., Joung, J., Slaymaker, I. M., Cox, D. B. T., Shmakov, S., Makarova, K. S., Semenova, E., Minakhin, L., Severinov, K., Regev, A., Lander, E. S., Koonin, E. V., and Zhang, F. 2016. C2c2 is a singl-component programmable RNA-guided RNA-targeting CRISPR effector. Science 353:aaf5573 [Epub ahead of print].

Adelman, Z. N., and Tu, Z. 2016. Control of mosquito-borne infectious diseases: Sex and gene drive. Trends Parasitol. 32:219-229.

Ai, T., Zhang, L., Gao, Z., Zhu, C. X., and Guo, X. 2011. Highly efficient virus resistance mediated by artificial microRNAs that target the suppressor of PVX and PVY in plants. Plant Biol. 13:304-316.

Ali, I., Amin, I., Briddon, R. W., and Mansoor, S. 2013. Artificial microRNAmediated resistance against the monopartite begomovirus Cotton leaf curl Burewala virus. Virol. J. 10:231.

Ali, Z., Abul-Faraj, A., Li, L., Ghosh, N., Piatek, M., Mahjoub, A., Aouida, M., Piatek, A., Baltes, N. J., Voytas, D. F., Dinesh-Kumar, S. P., and Mahfouz, M. M. 2015a. Efficient virus-mediated genome editing in plants using the CRISPR/Cas9 system. Mol. Plant 8:1288-1291.

Ali, Z., Abulfaraj, A., Idris, A., Ali, S., Tashkandi, M., and Mahfouz, M. M. 2015b. CRISPR/Cas9-mediated viral interference in plants. Genome Biol. $16: 238$.

Allen, E., and Howell, M. D. 2010. miRNAs in the biogenesis of trans-acting siRNAs in higher plants. Semin. Cell Dev. Biol. 21:798-804.

Anderson, P. K., Cunningham, A. A., Patel, N. G., Morales, F. J., Epstein, P. R., and Daszak, P. 2004. Emerging infectious diseases of plants: Pathogen pollution, climate change and agrotechnology drivers. Trends Ecol. Evol. 19:535-544.

Axtell, M. J., Jan, C., Rajagopalan, R., and Bartel, D. P. 2006. A two-hit trigger for siRNA biogenesis in plants. Cell 127:565-577.

Baltes, N. J., and Voytas, D. F. 2015. Enabling plant synthetic biology through genome engineering. Trends Biotechnol. 33:120-131.

Barkley, N. A., and Wang, M. L. 2008. Application of TILLING and EcoTILLING as reverse genetic approaches to elucidate the function of genes in plants and animals. Curr. Genomics 9:212-226.

Barrangou, R. 2015. The roles of CRISPR-Cas systems in adaptive immunity and beyond. Curr. Opin. Immunol. 32:36-41.

Barrangou, R., and Marraffini, L. A. 2014. CRISPR-Cas systems: Prokaryotes upgrade to adaptive immunity. Mol. Cell 54:234-244.

Baltes, N., Hummel, A., Konecna, E., Cegan, R., Bruns, A., Bisaro, D., and Voytas, D. F. 2015. Conferring resistance to geminiviruses with the CRISPR-Cas prokaryotic immune system. Nat. Plants 1:15145.

Baulcombe, D. 2005. RNA silencing. Trends Biochem. Sci. 30:290-293.

Baulcombe, D. C. 1996a. Mechanisms of pathogen-derived resistance to viruses in transgenic plants. Plant Cell 8:1833-1844.

Baulcombe, D. C. 1996b. RNA as a target and an initiator of post-transcriptional gene silencing in transgenic plants. Plant Mol. Biol. 32:79-88.

Beachy, R. N. 1999. Coat-protein-mediated resistance to tobacco mosaic virus: Discovery mechanisms and exploitation. Philos. T. R. Soc. B 354:659-664.

Belhaj, K., Chaparro-Garcia, A., Kamoun, S., Patron, N. J., and Nekrasov, V. 2015. Editing plant genomes with CRISPR/Cas9. Curr. Opin. Biotechnol. 32:76-84.

Bernstein, E., Caudy, A. A., Hammond, S. M., and Hannon, G. J. 2001. Role for a bidentate ribonuclease in the initiation step of RNA interference. Nature 409:363-366. 
Bhaya, D., Davison, M., and Barrangou, R. 2011. CRISPR-Cas systems in bacteria and archaea: Versatile small RNAs for adaptive defense and regulation. Annu. Rev. Genet. 45:273-297.

Boualem, A., Dogimont, C., and Bendahmane, A. 2016. The battle for survival between viruses and their host plants. Curr. Opin. Virol. 17:32-38.

Brodersen, P., and Voinnet, O. 2006. The diversity of RNA silencing pathways in plants. Trends Genet. 22:268-280.

Burt, A. 2003. Site-specific selfish genes as tools for the control and genetic engineering of natural populations. P. Roy. Soc. B-Biol. Sci. 270:921-928.

Burt, A. 2014. Heritable strategies for controlling insect vectors of disease. Philos. T. Roy. Soc. B 369:20130432.

Burt, A., and Trivers, R. 2009. Genes in conflict: The biology of selfish genetic elements. Harvard University Press, Cambridge, MA.

Castel, S. E., and Martienssen, R. A. 2013. RNA interference in the nucleus: Roles for small RNAs in transcription, epigenetics and beyond. Nat. Rev. Genet. 14:100-112.

Champer, J., Buchman, A., and Akbari, O. S. 2016. Cheating evolution: Engineering gene drives to manipulate the fate of wild populations. Nat. Rev. Genet. 17:146-159.

Chandrasegaran, S., and Carroll, D. 2016. Origins of programmable nucleases for genome engineering. J. Mol. Biol. 428:963-989.

Chandrasekaran, J., Brumin, M., Wolf, D., Leibman, D., Klap, C., Pearlsman, M., Sherman, A., Arazi, T., and Gal-On, A. 2016. Development of broad virus resistance in non-transgenic cucumber using CRISPR/Cas9 technology. Mol. Plant Pathol. doi:10.1111/mpp.12375. [Epub ahead of print].

Chaparro-Garcia, A., Kamoun, S., and Nekrasov, V. 2015. Boosting plant immunity with CRISPR/Cas. Genome Biol. 16:254.

Chen, H. M., Chen, L. T., Patel, K., Li, Y. H., Baulcombe, D. C., and Wu, S. H. 2010. 22-Nucleotide RNAs trigger secondary siRNA biogenesis in plants. Proc. Natl. Acad. Sci. U.S.A. 107:15269-15274.

Chen, L., Cheng, X., Cai, J., Zhan, L., Wu, X., Liu, Q., and Wu, X. 2016. Multiple virus resistance using artificial trans-acting siRNAs. J. Virol. Methods 228:16-20.

Cillo, F., and Palukaitis, P. 2014. Transgenic resistance. Adv. Virus Res. 90: 35-146.

Comai, L., Young, K., Till, B. J., Reynolds, S. H., Greene, E. A., Codomo, C. A., Enns, L.C., Johnson, J. E., Burtner, C., Odden, A. R., and Henikoff, S. 2004. Efficient discovery of DNA polymorphisms in natural populations by ecotilling. Plant J. 37:778-786.

Cong, L., Ran, F. A., Cox, D., Lin, S., Barretto, R., Habib, N. Hsu, P. D., Wu, X., Jiang, W., Marraffini, L. A.., and Zhang, F. 2013. Multiplex genome engineering using CRISPR/Cas systems. Science 339:819-823.

Cuperus, J. T., Carbonell, A., Fahlgren, N., Garcia-Ruiz, H., Burke, R. T., Takeda, A., Sullivan, C. M., Gilbert, S. D., Montgomery, T. A., and Carrington, J. C. 2010. Unique functionality of 22-nt miRNAs in triggering RDR6-dependent siRNA biogenesis from target transcripts in Arabidopsis. Nat. Struct. Mol. Biol. 17:997-1003.

Diaz-Pendon, J. A., Truniger, V., Nieto, C., Garcia-Mas, J., Bendahmane, A., and Aranda, M. A. 2004. Advances in understanding recessive resistance to plant viruses. Mol. Plant Pathol. 5:223-233.

Ding, S. W. 2010. RNA-based antiviral immunity. Nat. Rev. Immunol. 10: 632-644.

Ding, S. W., and Lu, R. 2011. Virus-derived siRNAs and piRNAs in immunity and pathogenesis. Curr. Opin. Virol. 1:533-544.

Ding, S. W., and Voinnet, O. 2007. Antiviral immunity directed by small RNAs. Cell 130:413-426.

Duan, C. G., Wang, C. H., Fang, R. X., and Guo, H. S. 2008. Artificial MicroRNAs highly accessible to targets confer efficient virus resistance in plants. J. Virol. 82:11084-11095.

Esvelt, K. M., Smidler, A. L., Catteruccia, F., and Church, G. M. 2014. Concerning RNA-guided gene drives for the alteration of wild populations. eLife doi:10.7554/eLife.03401. [Epub ahead of print]

Fahim, M., and Larkin, P. J. 2013. Designing effective amiRNA and multimeric amiRNA against plant viruses. Methods Mol. Biol. 942:357-377.

Fahim, M., Millar, A. A., Wood, C. C., and Larkin, P. J. 2012. Resistance to Wheat streak mosaic virus generated by expression of an artificial polycistronic microRNA in wheat. Plant Biotechnol. J. 10:150-163.

Fei, Q., Xia, R., and Meyers, B. C. 2013. Phased, secondary, small interfering RNAs in posttranscriptional regulatory networks. Plant Cell 25: 2400-2415.

Felippes, F. F., and Weigel, D. 2009. Triggering the formation of tasiRNAs in Arabidopsis thaliana: The role of microRNA miR173. EMBO (Eur. Mol. Biol. Organ.) Rep. 10:264-270.

Fu, Y., Foden, J. A., Khayter, C., Maeder, M. L., Reyon, D., Joung, J. K., and Sander, J. D. 2013. High-frequency off-target mutagenesis induced by CRISPR-Cas nucleases in human cells. Nat. Biotechnol. 31:822-826.

Fusaro, A. F., Matthew, L., Smith, N. A., Curtin, S. J., Dedic-Hagan, J., Ellacott, G. A., Watson, J. M., Wang, M. B., Brosnan, C., Carroll, B .J., and Waterhouse, P. M. 2006. RNA interference-inducing hairpin RNAs in plants act through the viral defence pathway. EMBO (Eur. Mol. Biol. Organ.) Rep. 7:1168-1175.

Galvez, L. C., Banerjee, J., Pinar, H., and Mitra, A. 2014. Engineered plant virus resistance. Plant Sci. 228:11-25.

Gantz, V. M., and Bier, E. 2015. Genome editing. The mutagenic chain reaction: A method for converting heterozygous to homozygous mutations. Science 348:442-444.

Gauffier, C., Lebaron, C., Moretti, A., Constant, C., Moquet, F., Bonnet, G., Caranta, C., and Gallois, J. L. 2016. A TILLING approach to generate broad-spectrum resistance to potyviruses in tomato is hampered by eIF4E gene redundancy. Plant J. 85:717-729.

Gelvin, S. B. 2003. Agrobacterium-mediated plant transformation: The biology behind the "gene-jockeying" tool. Microbiol. Mol. Biol. Rev. 67: 16-37.

Gonsalves, D. 1998. Control of papaya ringspot virus in papaya: A case study. Annu. Rev. Phytopathol. 36:415-437.

Guo, Y., Abernathy, B., Zeng, Y., and Ozias-Akins, P. 2015. TILLING by sequencing to identify induced mutations in stress resistance genes of peanut (Arachis hypogaea). BMC Genomics 16:157.

Hammond, A., Galizi, R., Kyrou, K., Simoni, A., Siniscalchi, C., Katsanos, D., Gribble, M., Baker, D., Marois, E., Russell, S., Burt, A., Windbichler, N., Crisanti, A., and Nolan, T. 2016. A CRISPR-Cas9 gene drive system targeting female reproduction in the malaria mosquito vector Anopheles gambiae. Nat. Biotechnol. 34:78-83.

Haurwitz, R. E., Jinek, M., Wiedenheft, B., Zhou, K., and Doudna, J. A. 2010. Sequence- and structure-specific RNA processing by a CRISPR endonuclease. Science 329:1355-1358

Henikoff, S., Till, B. J., and Comai, L. 2004. TILLING. Traditional mutagenesis meets functional genomics. Plant Physiol. 135:630-636.

Hull, R. 2009. Page 376 in: Comparative Plant Virology. Elsevier Academic Press, Amsterdam.

Ibiza, V., Canizares, J., and Nuez, F. 2010. EcoTILLING in Capsicum species: Searching for new virus resistances. BMC Genomics 11:631.

Jamal, M., Khan, F. A., Da, L., Habib, Z., Dai, J., and Cao, G. 2015. Keeping CRISPR/Cas on-target. Curr. Issues Mol. Biol. 20:1-20.

Jelly, N. S., Schellenbaum, P., Walter, B., and Maillot, P. 2012. Transient expression of artificial microRNAs targeting Grapevine fanleaf virus and evidence for RNA silencing in grapevine somatic embryos. Transgenic Res. 21:1319-1327.

Ji, X., Zhang, H., Zhang, Y., Wang, Y., and Gao, C. 2015. Establishing a CRISPR-Cas-like immune system conferring DNA virus resistance in plants. Nat. Plants 1:15144.

Jiang, J., and Laliberte, J. F. 2011. The genome-linked protein VPg of plant viruses-a protein with many partners. Curr. Opin. Virol. 1:347-354.

Jinek, M., Chylinski, K., Fonfara, I., Hauer, M., Doudna, J. A., and Charpentier, E. 2012. A programmable dual-RNA-guided DNA endonuclease in adaptive bacterial immunity. Science 337:816-821.

Jinek, M., East, A., Cheng, A., Lin, S., Ma, E., and Doudna, J. 2013. RNAprogrammed genome editing in human cells. eLife 2:e00471.

Kanchiswamy, C. N., Malnoy, M., Velasco, R., Kim, J. S., and Viola, R. 2015. Non-GMO genetically edited crop plants. Trends Biotechnol. 33:489-491.

Kennedy, E. M., and Cullen, B. R. 2015. Bacterial CRISPR/Cas DNA endonucleases: A revolutionary technology that could dramatically impact viral research and treatment. Virology 479-480:213-220.

Khraiwesh, B., Ossowski, S., Weigel, D., Reski, R., and Frank, W. 2008. Specific gene silencing by artificial MicroRNAs in Physcomitrella patens: An alternative to targeted gene knockouts. Plant Physiol. 148:684-693.

Kim, H., and Kim, J. S. 2014. A guide to genome engineering with programmable nucleases. Nat. Rev. Genet. 15:321-334.

Kis, A., Tholt, G., Ivanics, M., Varallyay, E., Jenes, B., and Havelda, Z. 2015. Polycistronic artificial miRNA-mediated resistance to Wheat dwarf virus in barley is highly efficient at low temperature. Mol. Plant Pathol. 17:427-437.

Kistler, K. E., Vosshall, L. B., and Matthews, B. J. 2015. Genome engineering with CRISPR-Cas9 in the mosquito Aedes aegypti. Cell Reports 11:51-60.

Kung, Y. J., Lin, S. S., Huang, Y. L., Chen, T. C., Harish, S. S., Chua, N. H., and Yeh, S. D. 2012. Multiple artificial microRNAs targeting conserved motifs of the replicase gene confer robust transgenic resistance to negativesense single-stranded RNA plant virus. Mol. Plant Pathol. 13:303-317.

Kurowska, M., Daszkowska-Golec, A., Gruszka, D., Marzec, M., Szurman, M., Szarejko, I., and Maluszynski, M. 2011. TILLING: A shortcut in functional genomics. J. Appl. Genet. 52:371-390.

Lapidot, M., Gafny, R., Ding, B., Wolf, S., Lucas, W. J., and Beachy, R. N. 1993. A dysfunc-tional movement protein of tobacco mosaic virus that partially modifies theplasmodesmata and limits virus spread in transgenic plants. Plant J. 4:959-970.

Lin, S. S., Wu, H. W., Elena, S. F., Chen, K. C., Niu, Q. W., Yeh, S. D., Chen, C. C., and Chua, N. H. 2009. Molecular evolution of a viral non-coding sequence under the selective pressure of amiRNA-mediated silencing. PLoS Pathog. 5:e1000312. 
Lindbo, J. A., and Dougherty, W. G. 1992a. Pathogen-derived resistance to a potyvirus: Immune and resistant phenotypes in transgenic tobacco expressing altered forms of a potyvirus coat protein nucleotide sequence. Mol. Plant-Microbe Interact. 5:144-153.

Lindbo, J. A., and Dougherty, W. G. 1992b. Untranslatable transcripts of the tobacco etch virus coat protein gene sequence can interfere with tobacco etch virus replication in transgenic plants and protoplasts. Virology 189: 725-733.

Lindbo, J. A., Silva-Rosales, L., Proebsting, W. M., and Dougherty, W. G. 1993. Induction of a highly specific antiviral state in transgenic plants: Implications for regulation of gene expression and virus resistance. Plant Cell 5:1749-1759.

Liu, D., Hu, R., Palla, K. J., Tuskan, G. A., and Yang, X. 2016. Advances and perspectives on the use of CRISPR/Cas9 systems in plant genomics research. Curr. Opin. Plant Biol. 30:70-77.

Mali, P., Aach, J., Stranges, P. B., Esvelt, K. M., Moosburner, M., Kosuri, S., Yang, L., and Church, G. M. 2013. CAS9 transcriptional activators for target specificity screening and paired nickases for cooperative genome engineering. Nat. Biotechnol. 31:833-838.

Marshall, J. M., and Hay, B. A. 2012. General principles of single-construct chromosomal gene drive. Evolution 66:2150-2166.

Martínez, F., Elena, S. F., and Daros, J. A. 2013. Fate of artificial microRNAmediated resistance to plant viruses in mixed infections. Phytopathol 103: 870-876

McCallum, C. M., Comai, L., Greene, E. A., and Henikoff, S. 2000a. Targeted screening for induced mutations. Nat. Biotechnol. 18:455-457.

McCallum, C. M., Comai, L., Greene, E. A., and Henikoff, S. 2000b. Targeting induced local lesions in genomes (TILLING) for plant functional genomics. Plant Physiol. 123:439-442.

Menda, N., Semel, Y., Peled, D., Eshed, Y., and Zamir, D. 2004. In silico screening of a saturated mutation library of tomato. Plant J. 38:861-872.

Mitter, N., Zhai, Y., Bai, A. X., Chua, K., Eid, S., Constantin, M., Mitchell, R., and Pappu, H. R. 2016. Evaluation and identification of candidate genes for artificial microRNA-mediated resistance to tomato spotted wilt virus. Virus Res. 211:151-158.

Nieto, C., Piron, F., Dalmais, M., Marco, C. F., Moriones, E., GomezGuillamon, M. L., Truniger, V., Gómez, P., Garcia-Mas, J., Aranda, M. A., Bendahmane, A. 2007. EcoTILLING for the identification of allelic variants of melon eIF4E, a factor that controls virus susceptibility. BMC Plant Biol. $7: 34$

Niu, Q. W., Lin, S. S., Reyes, J. L., Chen, K. C., Wu, H. W., Yeh, S. D., and Chua, N. H. 2006. Expression of artificial microRNAs in transgenic Arabidopsis thaliana confers virus resistance. Nat. Biotechnol. 24: 1420-1428.

Oleykowski, C. A., Bronson Mullins, C. R., Godwin, A. K., and Yeung, A. T. 1998. Mutation detection using a novel plant endonuclease. Nucleic Acids Res. 26:4597-4602.

Ossowski, S., Schwab, R., and Weigel, D. 2008. Gene silencing in plants using artificial microRNAs and other small RNAs. Plant J. 53:674-690.

Piron, F., Nicolai, M., Minoia, S., Piednoir, E., Moretti, A., Salgues, A., Zamir, D., Caranta, C., and Bendahmane, A. 2010. An induced mutation in tomato eIF4E leads to immunity to two potyviruses. PLoS One 5: e11313.

Popp, J., and Hantos, K. 2011. The impact of crop protection on agricultural production. In: Studies in Agricultural Economics, Vol. 113. Published online. http://EconPapers.repec.org/RePEc:ags:stagec:102401

Price, A. A., Sampson, T. R., Ratner, H. K., Grakoui, A., and Weiss, D. S. 2015. Cas9-mediated targeting of viral RNA in eukaryotic cells. Proc. Natl. Acad. Sci. U.S.A. 112:6164-6169.

Pyott, D. D., Sheehan, E., and Molnar, A. 2016. Engineering of CRISPR/Cas9mediated potyvirus resistance in transgene-free Arabidopsis plants. Mol. Plant Pathol. doi:10.1111/mpp.12417. [Epub ahead of print].

Qu, J., Ye, J., and Fang, R. 2007. Artificial microRNA-mediated virus resistance in plants. J. Virol. 81:6690-6699.

Ran, F. A., Hsu, P. D., Lin, C. Y., Gootenberg, J. S., Konermann, S., Trevino, A. E., Scott, D. A., Inoue, A., Matoba, S., Zhang, Y., and Zhang, F. 2013. Double nicking by RNA-guided CRISPR Cas9 for enhanced genome editing specificity. Cell 154:1380-1389.

Robaglia, C., and Caranta, C. 2006. Translation initiation factors: A weak link in plant RNA virus infection. Trends Plant Sci. 11:40-45.

Sander, J. D., and Joung, J. K. 2014. CRISPR-Cas systems for editing, regulating and targeting genomes. Nat. Biotechnol. 32:347-355.

Schwab, R., Ossowski, S., Riester, M., Warthmann, N., and Weigel, D. 2006. Highly specific gene silencing by artificial microRNAs in Arabidopsis. Plant Cell 18:1121-1133.

Singh, A., Taneja, J., Dasgupta, I., and Mukherjee, S. K. 2015. Development of plants resistant to tomato geminiviruses using artificial trans-acting small interfering RNA. Mol. Plant Pathol. 16:724-734.
Song, Y. Z., Han, Q. J., Jiang, F., Sun, R. Z., Fan, Z. H., Zhu, C. X., and Wen, F. J. 2014. Effects of the sequence characteristics of miRNAs on multi-viral resistance mediated by single amiRNAs in transgenic tobacco. Plant Physiol. Biochem. 77:90-98

Sternberg, S. H., and Doudna, J. A. 2015. Expanding the Biologist's Toolkit with CRISPR-Cas9. Mol. Cell 58:568-574.

Szittya, G., Silhavy, D., Molnar, A., Havelda, Z., Lovas, A., Lakatos, L., Banfalvi, Z., and Burgyan, J. 2003. Low temperature inhibits RNA silencing-mediated defence by the control of siRNA generation. EMBO (Eur. Mol. Biol. Organ.) J. 22:633-640.

Teotia, S., Singh, D., Tang, X., and Tang, G. 2016. Essential RNA-based technologies and their applications in plant functional genomics. Trends Biotechnol. 34:106-123.

Till, B. J., Zerr, T., Comai, L., and Henikoff, S. 2006. A protocol for TILLING and ecotilling in plants and animals. Nat. Protoc. 1:2465-2477.

Truniger, V., and Aranda, M. A. 2009. Recessive resistance to plant viruses. Adv. Virus Res. 75:119-159, 231.

Truniger, V., Nieto, C., Gonzalez-Ibeas, D., and Aranda, M. 2008. Mechanism of plant eIF4E-mediated resistance against a Carmovirus (Tombusviridae): Cap-independent translation of a viral RNA controlled in cis by an (a) virulence determinant. Plant J. 56:716-727.

Tsai, H., Howell, T., Nitcher, R., Missirian, V., Watson, B., Ngo, K. J., Lieberman, M., Fass, J., Uauy, C., Tran, R. K., Khan, A. A., Filkov, V., Tai, T. H., Dubcovsky, J., and Comai, L. 2011. Discovery of rare mutations in populations: TILLING by sequencing. Plant Physiol. 156:1257-1268.

Tsai, H., Ngo, K., Lieberman, M., Missirian, V., and Comai, L. 2015. Tilling by sequencing. Methods Mol. Biol. 1284:359-380.

Ülker, B., and Weisshaar, B. 2010. Resources for reverse genetics approaches in Arabidopsis thaliana. Pages 527-560 in: Genetics and Genomics of the Brassicaceae. Plant Genetics and Genomics: Crops and Models Vol. 9. Springer, New York.

Valencia-Sanchez, M. A., Liu, J., Hannon, G. J., and Parker, R. 2006. Control of translation and mRNA degradation by miRNAs and siRNAs. Genes Dev. 20:515-524.

Vaucheret, H. 2006. Post-transcriptional small RNA pathways in plants: Mechanisms and regulations. Genes Dev. 20:759-771.

Voinnet, O. 2009. Origin, biogenesis, and activity of plant microRNAs. Cell 136:669-687.

Voytas, D. F. 2013. Plant genome engineering with sequence-specific nucleases. Annu. Rev. Plant Biol. 64:327-350.

Wagaba, H., Patil, B. L., Mukasa, S., Alicai, T., Fauquet, C. M., and Taylor, N. J. 2016. Artificial microRNA-derived resistance to Cassava brown streak disease. J. Virol. Methods 231:38-43.

Wang, N., and Shi, L. 2015. Screening of mutations by TILLING in plants. Methods Mol. Biol. 1245:193-203.

Waterhouse, P. M., Graham, M. W., and Wang, M. B. 1998. Virus resistance and gene silencing in plants can be induced by simultaneous expression of sense and antisense RNA. Proc. Natl. Acad. Sci. U.S.A. 95:13959-13964.

Waterhouse, P. M., Wang, M. B., and Lough, T. 2001. Gene silencing as an adaptive defence against viruses. Nature 411:834-842.

Wittmann, S., Chatel, H., Fortin, M. G., and Laliberte, J. F. 1997. Interaction of the viral protein genome linked of turnip mosaic potyvirus with the translational eukaryotic initiation factor (iso) 4E of Arabidopsis thaliana using the yeast two-hybrid system. Virology 234:84-92.

Woo, J. W., Kim, J., Kwon, S. I., Corvalan, C., Cho, S. W., Kim, H., Kim, S. G., Kim, S. T., Choe, S., and Kim, J. S. 2015. DNA-free genome editing in plants with preassembled CRISPR-Cas9 ribonucleoproteins. Nat. Biotechnol. 33:1162-1164.

Wright, A. V., Nunez, J. K., and Doudna, J. A. 2016. Biology and applications of CRISPR systems: Harnessing nature's toolbox for genome engineering. Cell 164:29-44.

Xie, K., Minkenberg, B., and Yang, Y. 2015a. Boosting CRISPR/Cas9 multiplex editing capability with the endogenous tRNA-processing system. Proc. Natl. Acad. Sci. U.S.A. 112:3570-3575.

Xie, M., Zhang, S., and Yu, B. 2015b. microRNA biogenesis, degradation and activity in plants. Cell. Mol. Life Sci. 72:87-99.

Yoshikawa, M. 2013. Biogenesis of trans-acting siRNAs, endogenous secondary siRNAs in plants. Genes Genet. Syst. 88:77-84.

Zaidi, S. S., Mansoor, S., Ali, Z., Tashkandi, M., and Mahfouz, M. M. 2016. Engineering plants for geminivirus resistance with CRISPR/Cas9 system. Trends Plant Sci. 21:279-281. doi:10.1016/j.tplants.2016.01.023. [Epub ahead of print]

Zhang, D., Li, Z., and Li, J. 2015. New antiviral weapon for plants. Nat. Plants $1: 14146$

Zhang, X., Li, H., Zhang, J., Zhang, C., Gong, P., Ziaf, K., Xiao, F., and Ye, Z. 2011. Expression of artificial microRNAs in tomato confers efficient and stable virus resistance in a cell-autonomous manner. Transgenic Res. 20:569-581. 